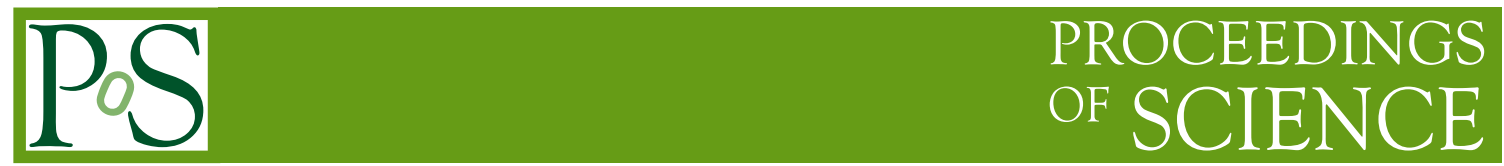

\title{
Noncommutative Geometry, the Spectral Action and Fundamental Symmetries
}

\author{
Fedele Lizzi* \\ Università di napoli Federico II \\ E-mail: fedele.lizzi@na.infn.it
}

\begin{abstract}
Noncommutative Geometry, i.e., the spectral data of generalized spaces, provides a fruitful approach to the standard model of fundamental interactions. This is done via the spectral action, which is a function of the Dirac operator. It is a regularized trace, cutoff at a scale. This cutoff is stye point in which all gauge interactions are equally strong, and it may represent a phase transition of the theory to a pre geometric phase. I will discuss the role of this field theory cutoff, and the symmetries and structure of space-time that one can infer from the spectral action.
\end{abstract}

Frontiers of Fundamental Physics 14 - FFP14,

15-18 July 2014

Aix Marseille University (AMU) Saint-Charles Campus, Marseille

\footnotetext{
*Speaker.
} 